\title{
The efficacy and safety of quantitative flow ratio-guided complete revascularization in patients with ST-segment elevation myocardial infarction and multivessel disease: A pilot randomized controlled trial
}

\author{
Jing Zhang ${ }^{1,2 \#}$, Mingyan Yao ${ }^{3 \#}$, Xinwei Jia ${ }^{1 *}$, Huiping Feng ${ }^{1}$,
} Jingjing $\mathrm{Fu}^{4}$, Wei Tang ${ }^{1}$, Hongliang Cong ${ }^{2 *}$

${ }^{1}$ Department of Cardiology, Affiliated Hospital of Hebei University, Baoding, China ${ }^{2}$ Department of Cardiology, Thoracic Clinical College, Tianjin Medical University, Tianjin, China

${ }^{3}$ Department of Endocrinology, Baoding No. 1 Central Hospital, Baoding, China

${ }^{4}$ Department of Cardiology, Fengfeng General Hospital, North China Medical and Health Group, Handan, China

\begin{abstract}
Background: In patients with ST-segment elevation myocardial infarction (STEMI) and multivessel disease (MVD), the treatment strategy for non-infarct-related artery (non-IRA) remains controversial. Quantitative flow ratio (QFR) is a new angiography-based physiological assessment index. However, there is little evidence on the practical clinical application of QFR.

Methods: Two hundred and twenty-nine patients with STEMI and MVD were recruited for this study. Patients were randomly assigned to either receive QFR-guided complete revascularization ( $Q F R-G-C R$ ) of non-IRA or receive no further invasive treatment. The primary $\left(1^{\circ}\right)$ endpoint analyzed included death due to all causes, non-fatal myocardial infarction (MI), and ischemia-induced revascularization at 12 months post-surgery. Secondary $\left(2^{\circ}\right)$ endpoints included cardiovascular death, unstable angina, stent thrombosis, New York Heart Association (NYHA) class IV heart failure, and stroke at 1 year post surgery. Massive bleeding and contrast-associated acute kidney injury (CAKI) were used as safety endpoints.

Results: Around the 12 month follow up, the $1^{\circ}$ outcome was recorded in 11/115 patients (9.6\%) in the QFR-G-CR population, relative to 23/114 patients (20.1\%) in the IRA-only PCI population (hazard ratio [HR]: 0.45; 95\% confidence interval [CI]: 0.22-0.92; $p=0.025)$. Unstable angina in $6(5.2 \%)$ and $16(14.0 \%$ ) patients (HR: 0.36; 95\% CI: 0.14-0.92; $p=0.026$ ), respectively. No marked alterations were found in the massive bleeding and CAKI categories.

Conclusions: In conclusion, STEMI and MVD patients can benefit from QFR-G-CR of non-IRA lesions in the initial stages of acute MI. This can help reduce incidences of major adverse cardiovascular events and unstable angina, relative to IRA treatment only.

Chinese Clinical Trial Registration number: ChiCTR2100044120. (Cardiol J 2023; 30, 2: 178-187)

Key words: ST-segment elevation myocardial infarction, multivessel disease, complete revascularization, physiological assessment, quantitative flow ratio
\end{abstract}

Address for correspondence: Xinwei Jia, MD, Department of Cardiology, Affiliated Hospital of Hebei University, No. 212 Yuhuadong Road, Baoding 071000, China. tel: +86-0312-5983865, fax: +86-0312-5983865, e-mail: xinweij@126.com; Hongliang Cong, MD, Department of Cardiology, Thoracic Clinical College, Tianjin Medical University, No. 261 Tai'erzhuang Road, Jinnan District, Tianjin 300222, China, tel: +86-022-88185111, fax: +86-022-88185111, e-mail: conghongliangmd@126.com

Received: 23.03.2021 Accepted: 27.08.2021 Early publication date: 23.09.2021

\#These authors contributed equally to this work.

*These authors also contributed equally to this work.

This article is available in open access under Creative Common Attribution-Non-Commercial-No Derivatives 4.0 International (CC BY-NC-ND 4.0) license, allowing to download articles and share them with others as long as they credit the authors and the publisher, but without permission to change them in any way or use them commercially. 


\section{Introduction}

Multiple studies have revealed that 30-50\% of patients with ST-elevation myocardial infarction (STEMI) exhibit additional severe stenotic lesions in the non-infarct-related artery (non-IRA) [1]. The recommended treatment for these patients is primary percutaneous coronary intervention (pPCI) for infarct-related artery (IRA) [2]. The importance of revascularization during $\mathrm{pPCI}$ for clinically important stenoses of non-IRA is controversial. Prior studies have demonstrated that $\mathrm{pPCI}$ in non-IRA can be detrimental [3, 4]. Therefore, recent approaches are more geared toward complete revascularization (CR) [5-8]. However, the most suitable timing and program of PCI for these patients is still a common dilemma [9].

Earlier studies have revealed the highly beneficial use of fractional flow reserve (FFR) guided PCI for positive long-term outcomes $[10,11]$. Therefore, expanding the application of physiological assessment of lesions, a noninvasive, economi$\mathrm{cal}$, and reliable tool to evaluate the functionality of non-IRA may be highly beneficial. The quantitative flow ratio (QFR) is an angiography-based procedure used to assess the extent of coronary stenosis, according to the three-dimensional quantitative coronary angiography (3D-QCA) and contrast frame counting. Recently, multiple studies have reported on the feasibility and accuracy of QFR in predicting stenosis [12-15]. In addition, trials have shown that QFR can also be used for the functional assessment of non-IRA in STEMI and multivessel disease (MVD) patients [16, 17]. Our goal for this trial was to examine the efficacy of QFR-guided CR (QFR-G-CR) during the acute phase, relative to no invasive treatment, in STEMI and MVD patients, with previous $\mathrm{pPCI}$ of IRA.

\section{Methods}

\section{Study design}

This is a researcher-instigated, prospective, randomized clinical trial. Our goal was to evaluate the clinical outcomes of (QFR-G-CR against IRA only revascularization in STEMI and MVD patients. Our hypothesis was that the QFR-G-CR procedure would reduce incidences of major adverse cardiovascular events (MACE) at 1 year, relative to the IRA only revascularization procedure, with optimal medication treatment strategy. We followed the Declaration of Helsinki and were approved by the ethics committee of the Affiliated Hospital of Hebei University (HDFY-KL-LL-2018-36). We also received informed agreement documents from all patients. Independent clinical research associates observed the trial and accumulated data.

\section{Participants}

We recruited adult STEMI patients, who showed eligibility and were set up for pPCI within 12 hours of symptom onset. According to the contemporary guidelines, patients with STEMI $>12 \mathrm{~h}$ of onset are also indicated for pPCI, if evidence of ischemia persists. All participates were hemodynamically stable after the pPCI procedure, and had $\geq 1$ lesion, with a percent diameter stenosis (DS\%) between $50 \%$ and $90 \%$ in $\geq 1$ non-IRAs, with a $>2.0 \mathrm{~mm}$ standard vessel diameter by visual estimation or quantitative coronary angiography.

Patients with any of the criteria listed below were excluded from the study: (1) severe heart failure (HF) or cardiogenic shock (New York Heart Association [NYHA] $\geq$ III); (2) strongly weakened kidney function: creatinine $>150 \mu \mathrm{mol} / \mathrm{L}$ or glomerular filtration rate $<45 \mathrm{~mL} / \mathrm{kg} / 1.73 \mathrm{~m}^{2}$; (3) left main coronary artery disease; (4) chronic total occlusion; (5) allergic to contrast media or of relevant anticoagulants (unfraction heparin, bivalirudin and fondaparinux) or antiplatelet drugs (acetylsalicylic acid, clopidogrel and ticagrelor); (6) severe stenosis (DS\% > 90\%) with a Thrombolysis in Myocardial Infarction flow grade $\leq 2$ in the non-IRA; (7) complications post IRA therapy; (8) severe valve dysregulation; (9) with prior coronary artery bypass grafting $(\mathrm{CABG})$; and (10) any interrogated vessel regarded as not conducive to QFR measurement.

\section{Randomization, treatment, and follow-up}

Following a successful pPCI of IRA, we randomly assigned eligible patients to a QFR-G-CR or an IRA-only revascularization cohort. Randomization was done by using a computer-processed random list. Please refer to Figure 1 for the randomized treatment and follow up examinations of patients. Given the risk of in-stent restenosis, drug-eluting stents were employed for all lesions. Patients, randomly picked for $\mathrm{CR}$, underwent $\mathrm{QFR}$ measurements of non-IRA lesions. In case of all non-IRA lesions with QFR $\leq 0.80$, PCI was conducted during patient's hospital stays, regardless of the presence of clinical symptoms. However, patients with QFR $>0.80$ in all examined vessels were not given PCI. Patients, in the IRA only revascularization patient population, received $\mathrm{pPCI}$ and no other invasive procedures. Optimal medical therapy, based on contemporary guidelines, was 


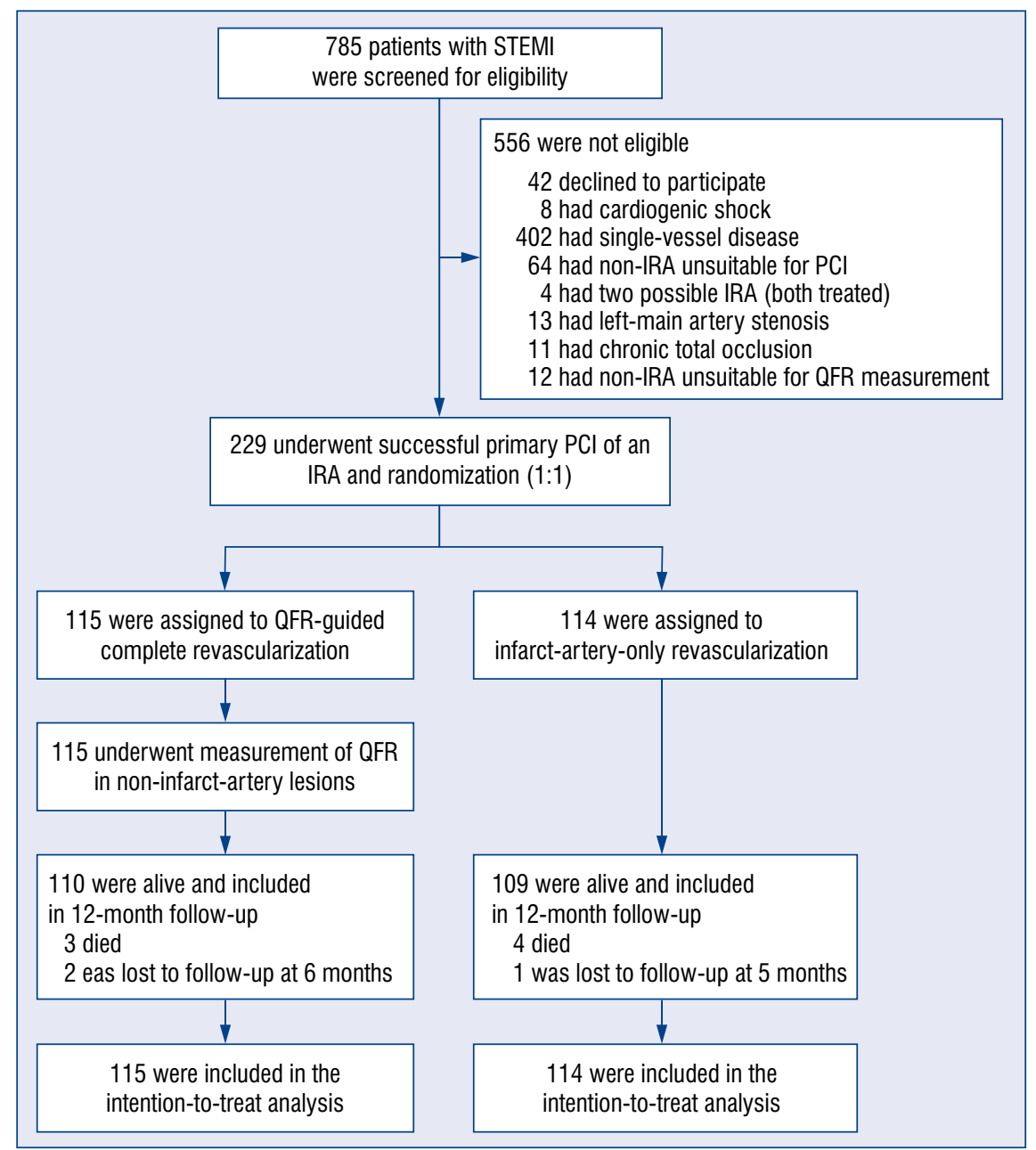

Figure 1. Enrollment, treatment, and follow-up. 229 patients with ST-segment elevation myocardial infarction (STEMI) and multivessel disease (MVD) were randomized to receive quantitative flow ratio (QFR)-guided complete revascularization (115) or infarct-related artery (IRA)-only percutaneous coronary intervention (PCI) (114). Patients were followedup for 1 year, and analysis was by intention-to-treat.

provided to both patient populations. Follow ups were performed once a month for up to 1 year post surgery.

\section{QFR measurement}

QFR computation was done offline, with the AngioPlus system (Pulse Medical Imaging Technology, Shanghai, China), as per routine operational directions [12]. Two independent, certified operators performed the QFR computation. QFR $\leq 0.80$ was used as the diagnostic cutoff value. We also performed QFR measurement after each surgery to analyze lesion correction. All angiogram files were stored in the core laboratory for further offline analysis.

\section{Endpoints}

The patients, in this study, were followed up till 1 year post surgery. The primary $\left(1^{\circ}\right)$ endpoint was the rate of MACE, which was a combination of patient death due to any reason, nonfatal MI, and ischemia-induced revascularization for the QFR-G-CR versus IRA-only patient populations. Secondary $\left(2^{\circ}\right)$ endpoints included the cardiovascular death, unstable angina, stent thrombosis, NYHA class IV HF, and stroke. Massive bleeding (BARC $\geq$ type 3 bleeding) [18] and contrast-associated acute kidney injury (CAKI) were used as safety endpoints. MI definition was the same as the fourth universal definition [19]. Ischemia-induced revascularization represented any repeat $\mathrm{pPCI}$ or 
Table 1. Patient demographic at baseline.

\begin{tabular}{|c|c|c|c|}
\hline Characteristic & $\begin{array}{l}\text { QFR-guided CR } \\
\qquad(n=115)\end{array}$ & $\begin{array}{l}\text { IRA-only PCI } \\
(\mathrm{n}=114)\end{array}$ & $\mathbf{P}$ \\
\hline Age [years] & $62.1 \pm 7.5$ & $62.7 \pm 6.2$ & 0.54 \\
\hline Male & $90(78.2 \%)$ & $91(79.8 \%)$ & 0.77 \\
\hline Hypertension & $59(51.3 \%)$ & $60(52.6 \%)$ & 0.84 \\
\hline Diabetes & $23(20.0 \%)$ & $21(18.4 \%)$ & 0.76 \\
\hline Hypercholesterolemia & $38(33.0 \%)$ & $36(31.2 \%)$ & 0.81 \\
\hline Current smoker & $56(48.6 \%)$ & $51(44.7 \%)$ & 0.55 \\
\hline Previous MI & $9(7.8 \%)$ & $8(7.0 \%)$ & 0.82 \\
\hline Previous $\mathrm{PCl}$ & $7(6.1 \%)$ & $6(5.3 \%)$ & 0.79 \\
\hline Previous stroke & $6(5.2 \%)$ & $3(2.6 \%)$ & $0.51 *$ \\
\hline \multicolumn{4}{|l|}{ Location of infarct ${ }^{* *}$ : } \\
\hline Anterior & $39(33.9 \%)$ & $37(32.5 \%)$ & 0.82 \\
\hline Inferior & $52(45.2 \%)$ & $55(48.2 \%)$ & 0.65 \\
\hline Posterior & $18(15.7 \%)$ & $16(14.0 \%)$ & 0.73 \\
\hline Lateral & $6(5.2 \%)$ & $6(5.3 \%)$ & 0.99 \\
\hline \multicolumn{4}{|c|}{ Symptom to balloon time [h]: } \\
\hline$<6$ & $53(46.1 \%)$ & $51(44.7 \%)$ & 0.84 \\
\hline $6-12$ & $49(42.6 \%)$ & $47(46.1 \%)$ & 0.83 \\
\hline$>12$ & $13(11.3 \%)$ & $16(13.9 \%)$ & 0.53 \\
\hline LDL-C [mmol/L] & $3.0 \pm 0.5$ & $2.9 \pm 0.4$ & 0.16 \\
\hline Peak creatinine $[\mu \mathrm{mol} / \mathrm{L}]$ & $74.3 \pm 13.7$ & $76.3 \pm 12.6$ & 0.27 \\
\hline \multicolumn{4}{|l|}{ Medications at discharge: } \\
\hline Acetylsalicylic acid & $115(100 \%)$ & $114(100 \%)$ & \\
\hline P2Y12 inhibitors: & $115(100 \%)$ & $114(100 \%)$ & \\
\hline ticagrelor & $82(71.3 \%)$ & $76(66.7 \%)$ & 0.45 \\
\hline clopidogrel & $33(28.7 \%)$ & $38(33.3 \%)$ & \\
\hline Beta-blocker & $107(93.0 \%)$ & $103(90.4 \%)$ & 0.46 \\
\hline ACEI or ARB & $110(95.7 \%)$ & $108(94.7 \%)$ & 0.75 \\
\hline Statin & $115(100 \%)$ & $114(100 \%)$ & \\
\hline
\end{tabular}

Data are shown as mean \pm standard deviation or number (\%). There were no significant differences between the two groups in any of the baseline characteristics. ${ }^{*} \mathrm{P}$ values were calculated with the use of a continuity-corrected chi-square test. ${ }^{*}$ The location of the infarct was determined on the basis of electrocardiography. ACEI — angiotensin converting enzyme inhibitor; ARB - angiotensin-II-receptor blocker; $\mathrm{CR}$ - complete revascularization; IRA — infarct-related artery; $\mathrm{MI}$ - myocardial infarction; LDL-C — low density lipoprotein cholesterol;

$\mathrm{PCl}$ - percutaneous coronary intervention; QFR - quantitative flow ratio

CABG, owing to constant chest pain, with or without electrocardiographic or biomarker alterations. Unstable angina was described as angina, even after appropriate therapy. Stent thrombosis was described as a stent site obstruction, in combination with acute myocardial ischemic manifestation, ischemic electrocardiographic alterations, or augmented myocardial enzymes levels. The definition of clinical events referred to the 2006 Academic Research Consortium (ARC) standards [20].

\section{Statistical analysis}

All outcomes from recruited patients were analyzed on an intent-to-treat approach. The $1^{\circ}$ outcomes were analyzed with the time-to-first-event approach. Categorical data, as clinical event rates, are presented as numbers and percentages (\%). Continuous data, evaluated with unpaired t-test, are presented as means \pm standard deviations (SD) for evenly distributed variables and, assessed with Mann-Whitney $\mathrm{U}$ test, and presented as medians 
Table 2. Procedural information.

\begin{tabular}{|c|c|c|c|}
\hline Characteristic & $\begin{array}{l}\text { QFR-guided CR } \\
\quad(n=115)\end{array}$ & $\begin{array}{l}\text { IRA-only PCI } \\
\quad(n=114)\end{array}$ & $\mathbf{P}$ \\
\hline 2-vessel disease & $86(74.8 \%)$ & $83(72.8 \%)$ & 0.73 \\
\hline 3-vessel disease & $29(25.2 \%)$ & $31(27.2 \%)$ & \\
\hline \multicolumn{4}{|l|}{ QFR-guided strategy: } \\
\hline QFR procedure successful in non-IRA: & $115(100 \%)$ & NA & \\
\hline non-IRA Lesions with QFR $\leq 0.8$ & $68(59.1 \%)$ & NA & \\
\hline non-IRA Lesions with QFR > 0.8 & $47(40.9 \%)$ & NA & \\
\hline Mean QFR value & $0.76 \pm 0.11$ & NA & \\
\hline Non-IRA Lesions successfully treated & $68 / 68(100 \%)$ & NA & \\
\hline During $\mathrm{pPCl}$ procedure & $42(61.8 \%)$ & NA & \\
\hline Early delayed $(\leq 7 d)$ & $26(38.2 \%)$ & NA & \\
\hline \multicolumn{4}{|l|}{ Treatment method: } \\
\hline Drug-eluting stent & $115(100 \%)$ & $113(99.1 \%)$ & $0.50^{*}$ \\
\hline Balloon dilation only & 0 & $1(0.9 \%)$ & \\
\hline No. of stents used per patient & $2(1-5)$ & $1(0-3)$ & $<0.001 * *$ \\
\hline Procedure time during $\mathrm{pPCl}[\mathrm{min}]$ & $63(40-132)$ & 49 (22-98) & $<0.001 * *$ \\
\hline Volume of contrast agent used during $\mathrm{pPCl}[\mathrm{mL}]$ & $195(120-400)$ & $158(70-315)$ & $<0.001^{* *}$ \\
\hline Radial access & $109(94.8 \%)$ & $106(93.0 \%)$ & 0.57 \\
\hline Thrombus aspiration & $38(33.0 \%)$ & $35(30.7 \%)$ & 0.70 \\
\hline
\end{tabular}

Data are shown as mean \pm standard deviation or number $(\%)$ or median (interquartile range). ${ }^{*} \mathrm{P}$ values were calculated with the use of a Fisher's exact test. * $P$ values were calculated with the use of a Mann-Whitney $U$ test. $C R$ - complete revascularization; IRA - infarct-related artery; non-IRA — non-infarct-related artery; $\mathrm{pPCl}$ — primary percutaneous coronary intervention; QFR — quantitative flow ratio; NA — not available

Table 3. Clinical and safety endpoints at the 1 year follow up.

\begin{tabular}{|c|c|c|c|c|}
\hline Characteristic & $\begin{array}{l}\text { QFR-guided CR } \\
\quad(n=115)\end{array}$ & $\begin{array}{l}\text { IRA-only PCI } \\
(\mathrm{n}=114)\end{array}$ & $\begin{array}{l}\text { Hazard ratio } \\
(95 \% \mathrm{Cl})\end{array}$ & $\mathbf{P}$ \\
\hline \multicolumn{5}{|l|}{ Primary endpoint } \\
\hline MACE (any first event)* & $11(9.6 \%)$ & $23(20.1 \%)$ & $0.45(0.22-0.92)$ & 0.025 \\
\hline All-cause mortality & $3(2.6 \%)$ & $4(3.5 \%)$ & $0.74(0.17-3.30)$ & 0.69 \\
\hline Nonfatal myocardial infarction & $3(2.6 \%)$ & $5(4.4 \%)$ & $0.58(0.14-2.44)$ & 0.47 \\
\hline Ischemia-driven revascularization & $8(7.0 \%)$ & $19(16.7 \%)$ & $0.40(0.18-0.91)$ & 0.024 \\
\hline $\mathrm{PCl}$ & $8(7.0 \%)$ & $18(15.8 \%)$ & $0.42(0.18-0.97)$ & 0.037 \\
\hline Coronary artery bypass graft & 0 & $1(0.9 \%)$ & NA & NA \\
\hline \multicolumn{5}{|l|}{ Secondary endpoints } \\
\hline Cardiovascular death & $2(1.7 \%)$ & $2(1.8 \%)$ & $0.99(0.14-7.01)$ & 0.99 \\
\hline Unstable angina & $6(5.2 \%)$ & $16(14.0 \%)$ & $0.36(0.14-0.92)$ & 0.026 \\
\hline Stent thrombosis & $1(0.9 \%)$ & $1(0.9 \%)$ & $0.99(0.06-15.78)$ & 0.99 \\
\hline NYHA class IV heart failure & $4(3.5 \%)$ & $5(4.4 \%)$ & $0.78(0.21-2.91)$ & 0.71 \\
\hline Stroke & 0 & $1(0.9 \%)$ & NA & NA \\
\hline \multicolumn{5}{|l|}{ Safety endpoints } \\
\hline Major bleedings & $3(2.6 \%)$ & $2(1.8 \%)$ & $1.48(0.25-8.88)$ & 0.66 \\
\hline Contrast-associated acute kidney injury & $2(1.7 \%)$ & $1(0.9 \%)$ & $1.99(0.18-21.93)$ & 0.57 \\
\hline
\end{tabular}

Values are number (\%) for occurrences of both first events and total events. *MACE denotes the composite of all-cause mortality, non-fatal myocardial infarction, and any ischemia-driven revascularization. $\mathrm{Cl}$ - confidence interval; $\mathrm{CR}$ - complete revascularization; IRA - infarctrelated artery; MACE — major adverse cardiovascular events; NYHA - New York Heart Association; PCl — percutaneous coronary intervention; QFR - quantitative flow ratio; NA — not available 


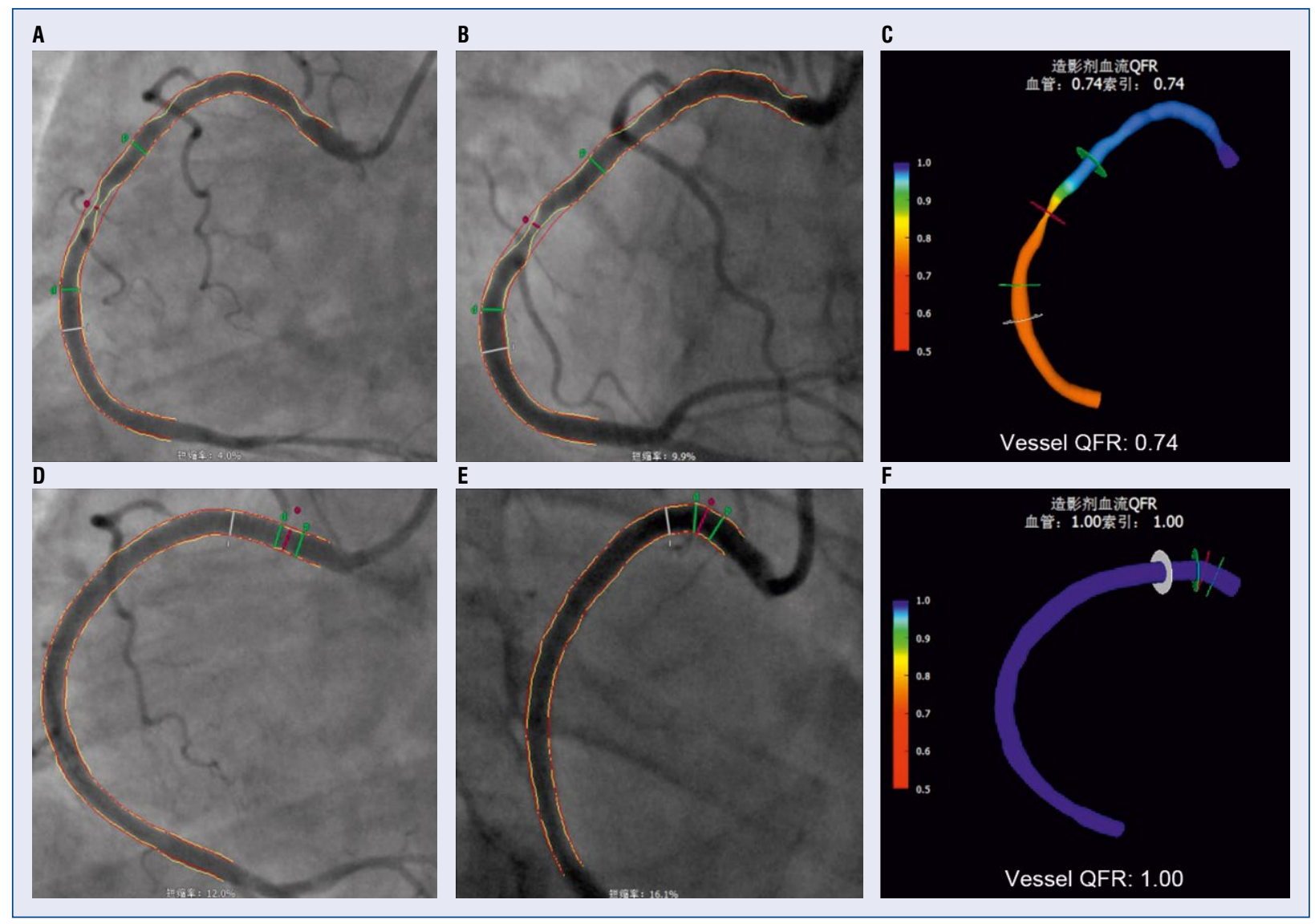

Figure 2. Example of quantitative flow ratio (QFR) computation in a right coronary artery (RCA). Example of assessment with QFR of a non-infarct-related artery (IRA) lesion; A, B. Non-IRA lesion in the mid-portion of RCA before percutaneous coronary intervention (PCI); C. Vessel QFR of RCA lesion before PCI; D, E. Non-IRA lesion in the midportion of RCA after PCl; F. Vessel QFR of RCA lesion after PCl.

\pm minimum (min) and maximum (max) values for unevenly distributed variables. The $\chi^{2}$ test or the Fisher exact test was employed for the assessment of categorical data. A two-sided $\mathrm{p}$ value $<0.05$ was considered statistically significant. When evaluating the time-to-event endpoints, the log-rank test was used and the Kaplan-Meier technique was employed to depict survival probability. Cox proportional-hazard models were fitted to predict hazard ratios (HR) with $95 \%$ confidence intervals (CI) for treatment comparisons. All analyses were performed with SPSS, version 22.0 (SPSS).

\section{Results}

\section{Patients and baseline characteristics}

Between August, 2019, and January, 2020, 229 STEMI and MVD patients who received pPCI were recruited for this study. The patients were randomized and 115 were placed in the QFR-G-CR category, and 114 in the IRA-only PCI category. The median follow-up time was 12.5 months (interquartile range: $11.9-13.0$ ). It was followed by all but 3 patients ( 2 in QFR-G-CR and 1 in IRA-only PCI patient population) (Fig. 1). The baseline features and risk factors were relatively the same between the groups (Table 1).

\section{Procedural data and treatment}

Procedural information for both patient populations is listed in Table 2. The procedural time for the QFR-G-CR cohort, during PPCI, was $\sim 14$ min longer $(\mathrm{p}<0.001)$, with $37 \mathrm{~mL}$ more of the contrast agent volume used $(\mathrm{p}<0.001)$, relative to the IRA-only PCI cohort. The QFR-G-CR group used more stents per patient $(\mathrm{p}<0.001)$ by treating more lesions. The proportion of MVD, use of radial access, and thrombus aspiration remained relatively the same in both cohorts (Table 2). The only exception was 1 participant of the IRA-only 


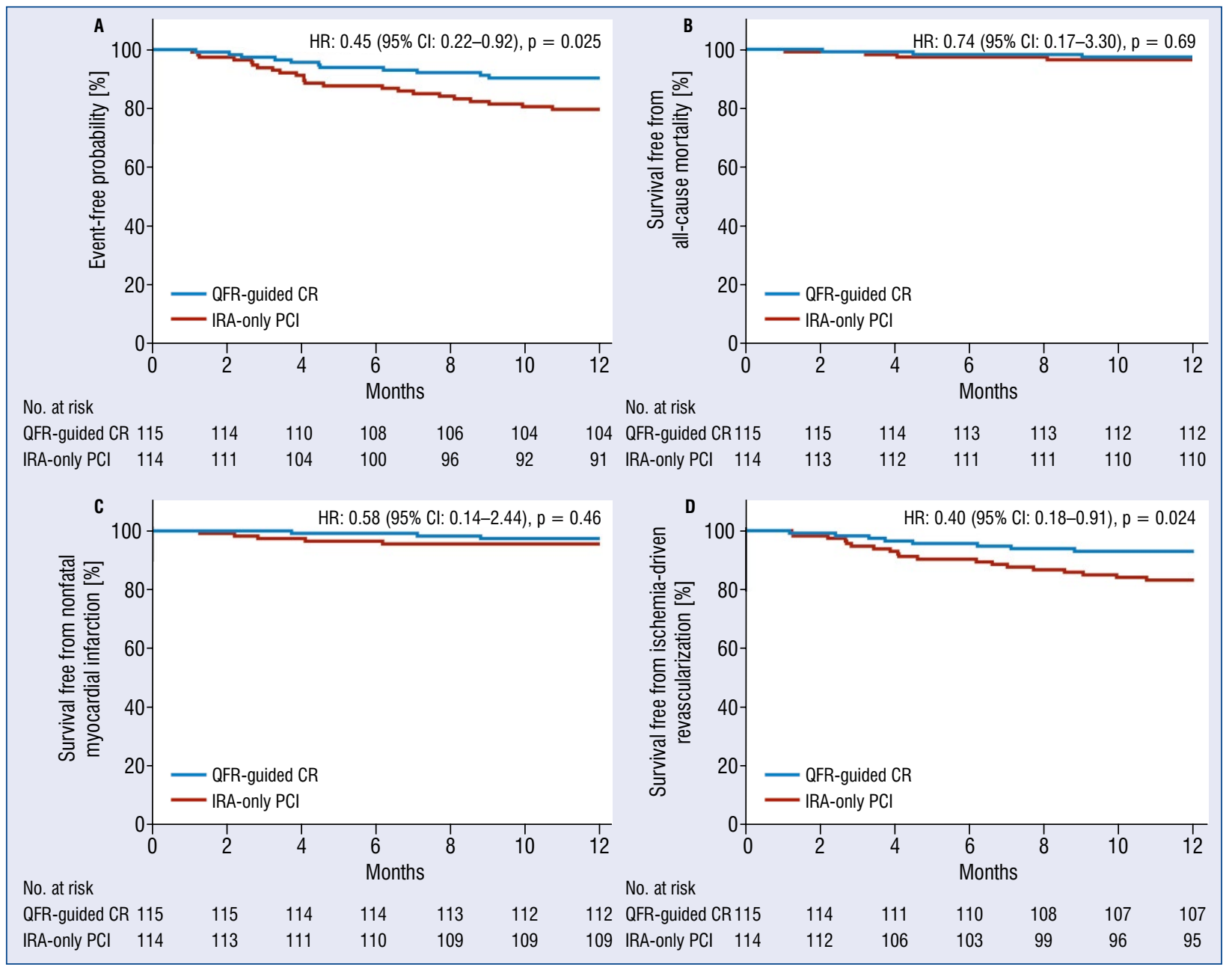

Figure 3. Kaplan-Meier curves for the primary $\left(1^{\circ}\right)$ endpoint and its components; A. Kaplan-Meier estimate of the survival rate of the $1^{\circ}$ endpoint (major adverse cardiovascular events [MACE]); B-D. The components of MACE (allcause mortality, nonfatal myocardial infarction, and ischemia-driven revascularization), respectively; $\mathrm{Cl}$ - confidence interval; $\mathrm{CR}$ - complete revascularization; HR - hazard ratio; IRA - infarct-related artery; $\mathrm{PCl}$ - percutaneous coronary intervention; QFR - quantitative flow ratio.

PCI cohort who received balloon dilation only. Both cohorts received the same management during discharge (Table 1).

\section{QFR-related endpoints}

In the QFR-G-CR group, QFR values were successfully measured in all lesions of non-IRA (Table 2 ). The mean $\mathrm{QFR}$ value was $0.76 \pm 0.11$. Of the 115 patients allocated in this group, $68(59.1 \%)$ had QFR values for one or more lesions in non-IRA that were less than or equal to the discrimination value of 0.80 . These lesions were chosen for stent placement (Fig. 2). In 42 (61.8\%) of these 68 patients, additional PCI were performed during the pPCI procedure; the rest (26 patients, $38.2 \%$ ) received early delayed PCI during the index admission ( $\leq 7$ days).

\section{First endpoints}

The $1^{\circ}$ endpoint (MACE) was observed in 11 (9.6\%) patients in the QFR-G-CR cohort, relative to $23(20.1 \%)$ patients in the IRA-only PCI cohort at the 1-year follow-up (HR: 0.45; 95\% CI: 0.22-0.92; $\mathrm{p}=0.024$ ) (Table 3, Fig. 3A). The Kaplan-Meier curves diverged early, and remained separated at the 1-year follow-up (Fig. 3A). The $1^{\circ}$ endpoint curves are presented in Figure 3B-D. The result was driven mainly by the higher incidence of ischemia-driven revascularization performed in the latter group ( $7.2 \%$ vs. $16.7 \%$; HR: $0.40 ; 95 \% \mathrm{CI}$ : $0.18-0.91 ; \mathrm{p}=0.024)$. Therefore, $\mathrm{QFR}-\mathrm{G}-\mathrm{CR}$ fared much better, compared to IRA-only revascularization, in that it produced a whopping $60 \%$ decrease in repeat revascularizations. 


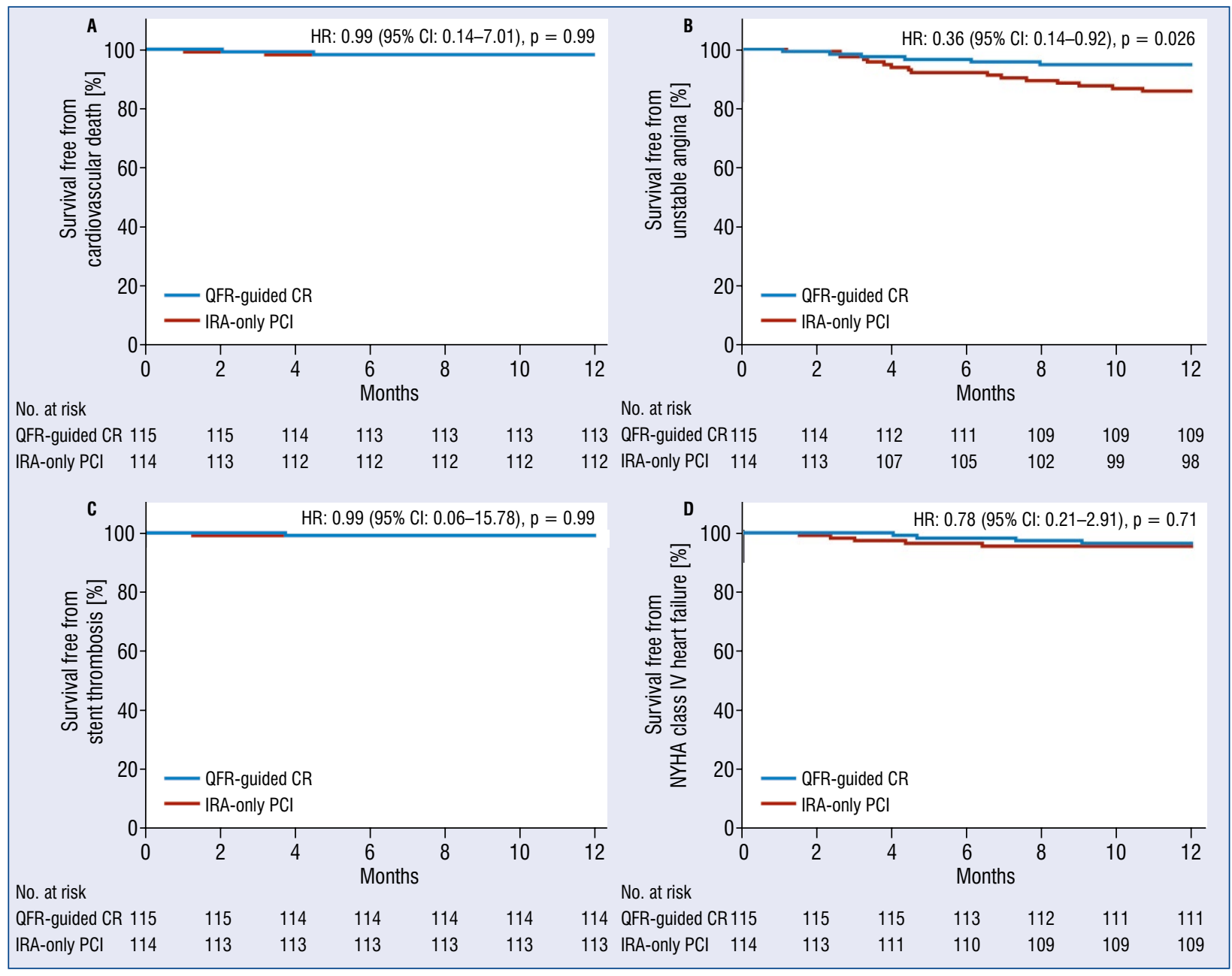

Figure 4. Kaplan-Meier curves for the secondary $\left(2^{\circ}\right)$ endpoints; A-D. Kaplan-Meier estimates of the survival rate of the components of $2^{\circ}$ endpoint (cardiovascular death, unstable angina, stent thrombosis, and New York Heart Association class IV heart failure), respectively; $\mathrm{Cl}$ - confidence interval; $\mathrm{CR}$ - complete revascularization; HR - hazard ratio; IRA — infarct-related artery; PCl - percutaneous coronary intervention; QFR - quantitative flow ratio.

\section{Second and safety endpoints}

Unstable angina event rate was observed in $6(5.2 \%)$ patients in the QFR-G-CR cohort, relative to $16(14.0 \%)$ patients in the IRA only PCI cohort (HR: $0.36 ; 95 \%$ CI: 0.14-0.92; $\mathrm{p}=0.026$ ) (Table 3, Fig. 4B). Other $2^{\circ}$ endpoints in the two cohorts were not significantly different (Fig. 4A, C, D). Moreover, no significant difference was observed in massive bleeding and CAKI risk (Table 3).

\section{Discussion}

The present study demonstrated that supplementary QFR-G-CR of non-IRA lesions during or $<7$ days post pPCI in STEMI and MVD patients produced a dramatically reduced rate of primary endpoint. This was primarily due to eliminating the need for repeat revascularizations. These findings are similar to other publications $[6,7]$. Furthermore, in the $2^{\circ}$ endpoints, the incidence of unstable angina in the QFR-G-CR cohort was far less, compared to the IRA-only cohort, suggesting that the QFR-G-CR procedure can greatly improve clinical outcomes and quality of life of patients. Although the volume of the contrast agent and the procedural time, during $\mathrm{pPCI}$, in the QFR-G-CR cohort, were higher, as compared to the IRA-only cohort, no increase in other safety events, such as major bleeding or CAKI, was observed.

Previous studies demonstrated that non-IRA can be evaluated in STEMI with MVD patients during $\mathrm{pPCI}$, but adenosine during the FFR process may cause slow blood flow and spasm in IRA. QFR, an angiography-based physiological assessment tool, presents an excellent option for the use of functional-based coronary stenosis examination, 
preventing the risk and discomfort seen with pressure wires and adenosine [21]. Based on the results of this study, the QFR-G-CR group extended the operation time by an average of $14 \mathrm{~min}$, which we believe is acceptable for STEMI patients with stable hemodynamics. However, there still exists a lack of evidence on the practical clinical application of QFR, and this study fills that gap. In the present trial, $\mathrm{QFR}-\mathrm{G}-\mathrm{CR}$ reduced MACE, and the percentage of angiographically significant non-IRA lesions with a $40 \%$ QFR $>0.80$. This suggested that nearly half of the non-IRA lesions that coronary angiography considered significant were, in fact, not physiologically significant.

There is still controversy regarding the optimal timing of early CR. Previous clinical trials, and recent meta-analyses, vary in the $\mathrm{CR}$ timing [22-24]. The CR with multi-vessel PCI for MI (COMPLETE) trial [8], with a larger sample size, demonstrated that $\mathrm{CR}$ fared much better than primary lesion only $\mathrm{PCI}$, when performed within 45 days. In this trial, the QFR-G-CR for STEMI and MVD patients were performed during the acute phase of STEMI (during or within 7 days after pPCI). Based on our results, the composite outcome with good safety endpoints (no increase in major bleeding or CAKI) was only seen with the QFR-G-CR treatment strategy, but not with the IRA-only PCI

\section{Limitations of the study}

There were some limitations in this trial. This study could not meet blinding requirements, due to interventional treatment. Given the open-label design, there could be bias that clinicians more likely performed subsequent revascularization on the IRA-only cohort. Some anatomic factors, such as ostial lesion, diffused long lesion, or severe vessel tortuosity, were not suitable for QFR assessment. Therefore, this can lead to selective bias among patients. As such, trials with larger patient populations are needed in future to discern the effects of QFR-G-CR on these endpoints. Finally, the present trial was followed-up for 1 year, and to elucidate long-term outcomes, future trials with long-term follow-ups are needed.

\section{Conclusions}

In conclusion, among STEMI and MVD patients, the strategy of QFR-G-CR of non-IRA lesions in the early stages of acute MI could reduce the incidence of MACE and unstable angina, relative to IRA treatment alone. Additionally, herein showed no increased risk of massive bleeding or CAKI within 1 year when using QFR-G-CR.

\section{Acknowledgments}

We acknowledge support of the Natural Science Foundation of Hebei Province (H2021201024). We thank all of the patients for their agreement to participate in this study.

\section{Conflict of interest: None declared}

\section{References}

1. Park DW, Clare RM, Schulte PJ, et al. Extent, location, and clinical significance of non-infarct-related coronary artery disease among patients with ST-elevation myocardial infarction. JAMA. 2014; 312(19): 2019-2027, doi: 10.1001/jama.2014.15095, indexed in Pubmed: 25399277.

2. Wong GC, Welsford M, Ainsworth C, et al. 2019 Canadian Cardiovascular Society/Canadian Association of Interventional Cardiology Guidelines on the Acute Management of ST-Elevation Myocardial Infarction: Focused Update on Regionalization and Reperfusion. Can J Cardiol. 2019; 35(2): 107-132, doi: 10.1016/j. cjca.2018.11.031, indexed in Pubmed: 30760415.

3. O'Gara PT, Kushner FG, Ascheim DD, et al. 2013 ACCF/AHA guideline for the management of ST-elevation myocardial infarction: a report of the American College of Cardiology Foundation/ American Heart Association Task Force on Practice Guidelines. J Am Coll Cardiol. 2013; 61(4): e78-e7e140, doi: 10.1016/j. jacc.2012.11.019, indexed in Pubmed: 23256914.

4. Hannan EL, Samadashvili Z, Walford G, et al. Culprit vessel percutaneous coronary intervention versus multivessel and staged percutaneous coronary intervention for ST-segment elevation myocardial infarction patients with multivessel disease. JACC Cardiovasc Interv. 2010; 3(1): 22-31, doi: 10.1016/j. jcin.2009.10.017, indexed in Pubmed: 20129564.

5. Gershlick AH, Khan JN, Kelly DJ, et al. Randomized trial of complete versus lesion-only revascularization in patients undergoing primary percutaneous coronary intervention for STEMI and multivessel disease: the CvLPRIT trial. J Am Coll Cardiol. 2015; 65(10): 963-972, doi: 10.1016/j.jacc.2014.12.038, indexed in Pubmed: 25766941.

6. Engstrøm T, Kelbæk H, Helqvist S, et al. Complete revascularisation versus treatment of the culprit lesion only in patients with ST-segment elevation myocardial infarction and multivessel disease (DANAMI-3-PRIMULTI): an open-label, randomised controlled trial. Lancet. 2015; 386(9994): 665-671, doi: 10.1016/ s0140-6736(15)60648-1.

7. Smits PC, Abdel-Wahab M, Neumann FJ, et al. Fractional flow reserve-guided multivessel angioplasty in myocardial infarction. N Engl J Med. 2017; 376(13): 1234-1244, doi: 10.1056/NEJMoa1701067, indexed in Pubmed: 28317428.

8. Mehta SR, Wood DA, Storey RF, et al. Complete revascularization with multivessel PCI for myocardial infarction. N Engl J Med. 2019; 381(15): 1411-1421, doi: 10.1056/NEJMoa1907775, indexed in Pubmed: 31475795.

9. Wood DA, Cairns JA, Mehta SR. Multivessel revascularization and ST-segment-elevation myocardial infarction: do we have the complete answer? Circ Cardiovasc Interv. 2017; 10(4), doi: 10.1161/CIRCINTERVENTIONS.117.005215, indexed in Pubmed: 28404625 . 
10. Xaplanteris P, Fournier S, Pijls NHJ, et al. Five-Year outcomes with PCI guided by fractional flow reserve. N Engl J Med. 2018; 379(3): 250-259, doi: 10.1056/NEJMoa1803538, indexed in Pubmed: 29785878.

11. Zhang D, Lv S, Song X, et al. Fractional flow reserve versus angiography for guiding percutaneous coronary intervention: a meta-analysis. Heart. 2015; 101(6): 455-462, doi: 10.1136/ heartinl-2014-306578, indexed in Pubmed: 25637372.

12. Tu S, Westra J, Yang J, et al. Diagnostic accuracy of fast computational approaches to derive fractional flow reserve from diagnostic coronary angiography: the international multicenter FAVOR pilot study. JACC Cardiovasc Interv. 2016; 9(19): 2024-2035, doi: 10.1016/j.jcin.2016.07.013, indexed in Pubmed: 27712739.

13. Xu Bo, Tu S, Qiao S, et al. Diagnostic accuracy of angiography-based quantitative flow ratio measurements for online assessment of coronary stenosis. J Am Coll Cardiol. 2017; 70(25): 3077-3087, doi: 10.1016/j.jacc.2017.10.035, indexed in Pubmed: 29101020

14. Westra J, Andersen BK, Campo G, et al. Diagnostic performance of in-procedure angiography-derived quantitative flow reserve compared to pressure-derived fractional flow reserve: the FAVOR II Europe-Japan study. J Am Heart Assoc. 2018; 7(14), doi: 10.1161/JAHA.118.009603, indexed in Pubmed: 29980523.

15. Westra J, Tu S, Winther S, et al. Evaluation of coronary artery stenosis by quantitative flow ratio during invasive coronary angiography: the WIFI II study (wire-free functional imaging II). Circ Cardiovasc Imaging. 2018; 11(3): e007107, doi: 10.1161/ CIRCIMAGING.117.007107, indexed in Pubmed: 29555835.

16. Spitaleri G, Tebaldi M, Biscaglia S, et al. Quantitative flow ratio identifies nonculprit coronary lesions requiring revascularization in patients with st-segment-elevation myocardial infarction and multivessel disease. Circ Cardiovasc Interv. 2018; 11(2): e006023, doi: 10.1161/CIRCINTERVENTIONS.117.006023, indexed in Pubmed: 29449325.

17. Sejr-Hansen M, Westra J, Thim T, et al. Quantitative flow ratio for immediate assessment of nonculprit lesions in patients with ST-segment elevation myocardial infarction: an iSTEMI substudy. Catheter Cardiovasc Interv. 2019; 94(5): 686-692, doi: 10.1002/ccd.28208, indexed in Pubmed: 30912257.

18. Mehran R, Rao SV, Bhatt DL, et al. Standardized bleeding definitions for cardiovascular clinical trials: a consensus report from the Bleeding Academic Research Consortium. Circulation. 2011; 123(23): 2736-2747, doi: 10.1161/CIRCULATIONAHA.110.009449, indexed in Pubmed: 21670242.

19. Thygesen K, Alpert JS, Jaffe AS, et al. Executive Group on behalf of the Joint European Society of Cardiology (ESC)/American College of Cardiology (ACC)/American Heart Association (AHA)/ World Heart Federation (WHF) Task Force for the Universal Definition of Myocardial Infarction. Fourth Universal Definition of Myocardial Infarction (2018). Glob Heart. 2018; 13(4): 305-338, doi: 10.1016/j.gheart.2018.08.004, indexed in Pubmed: 30154043.

20. Cutlip DE, Windecker S, Mehran R, et al. Academic Research Consortium. Clinical end points in coronary stent trials: a case for standardized definitions. Circulation. 2007; 115(17): $2344-$ -2351, doi: 10.1161/CIRCULATIONAHA.106.685313, indexed in Pubmed: 17470709.

21. Cesaro A, Gragnano F, Di Girolamo D, et al. Functional assessment of coronary stenosis: an overview of available techniques. Is quantitative flow ratio a step to the future? Expert Rev Cardiovasc Ther. 2018; 16(12): 951-962, doi: 10.1080/14779072.2018.1540303, indexed in Pubmed: 30352515 .

22. Kim MC, Bae S, Ahn Y, et al. Benefit of a staged in-hospital revascularization strategy in hemodynamically stable patients with ST-segment elevation myocardial infarction and multivessel disease: Analyses by risk stratification. Catheter Cardiovasc Interv. 2021; 97(6): 1151-1159, doi: 10.1002/ccd.29062, indexed in Pubmed: 32569397.

23. Elgendy IY, Gad M, Elbadawi A, et al. Is complete revascularization for multivessel disease during primary percutaneous coronary intervention associated with lower cardiovascular mortality? An updated meta-analysis and trial sequential of randomized trials. Eur Heart J Qual Care Clin Outcomes. 2020; 6(4): 341-342, doi: 10.1093/ehjqcco/qcz067, indexed in Pubmed: 31977003.

24. Pasceri V, Patti G, Pelliccia F, et al. Complete revascularization during primary percutaneous coronary intervention reduces death and myocardial infarction in patients with multivessel disease: meta-analysis and meta-regression of randomized trials. JACC Cardiovasc Interv. 2018; 11(9): 833-843, doi: 10.1016/j. jcin.2018.02.028, indexed in Pubmed: 29747913. 